\title{
The Impact of Innovation in the Satellite Industry on the Telecommunications Services Market
}

\author{
Krasimir Terziev ${ }^{l}$, Dimitar Karastoyanov ${ }^{l}$ \\ ${ }^{I}$ Institute of Information and Communication Technologies, Sofia, Bulgaria \\ Emails:krterziev@gmail.com, dimikara@abv.bg
}

\begin{abstract}
The article analyses the role of the satellites in global communications. Different types of orbits and different types of satellites are described. The commercial start of low and medium orbit satellites is considered. The integration of some Low Earth Orbit (LEO) projects with Teleports is commented. Some significant LEO/MEO (Medium Earth Orbit) projects are cited. The impact of the satellite industry and the Teleport systems on the technological ecosystem is discussed. LEO satellites for monitoring the earth's surface are presented.
\end{abstract}

Keywords: LEO, Orbit, Satellite, Teleport.

\section{Introduction}

Satellite connections and networks play an important role in global communications, [1]. They are part of the global telecommunications network [2]. Their indisputable advantage is that they provide instant communication to the most remote and inaccessible places (no need to build cable infrastructure, but only one receiving and/or transmitting antenna), are not affected by natural disasters on the earth's surface (earthquakes, floods and others), provide connections and internet to the seas and oceans (standard cable networks cannot connect sea-going vessels). Telecommunication networks could be modelled by separate graph models that already exist. Using the unified model makes it possible to solve new classes of problems that cannot be solved using traditional systems [3], [4]. The existing infrastructure could be base for some innovative navigation systems [5]. Such systems could be used for adequate vehicle navigation in urban environments [6].

In the 21 st century, we see that the near-Earth space is inhabited by thousands of satellites of different types and in different orbits, [7]. The main types of orbits of 
the satellites are shown in Fig. 1 [8], where: GEO is Geostationary Orbit; MEO is Medium Earth Orbit; HEO is Highly Elliptical Orbit; LEO is Low Earth Orbit.

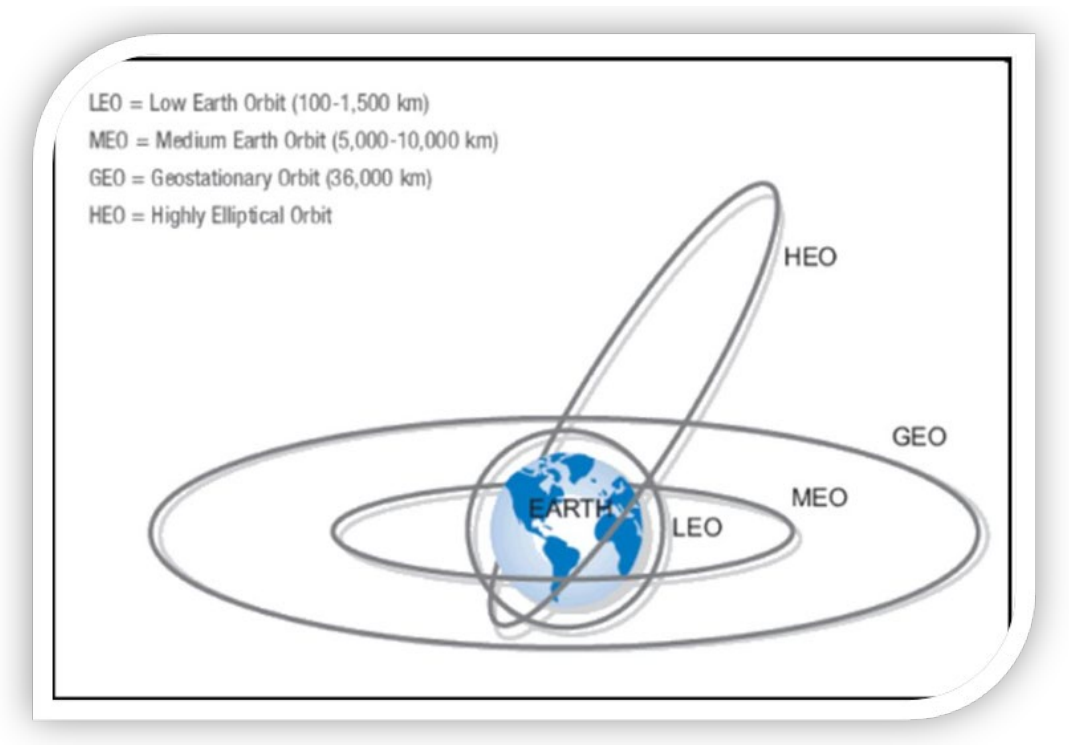

Fig. 1. Types of satellite orbits

Different types of satellites offer different types of telecommunication services as follows [9], [10]:

- FSS (Fixed Satellite Services) - stationary satellite services. Provide communications between one or more fixed ground points through retransmission.

- BSS (Broadcast Satellite Service) - Radio and Television broadcasting this technology is used in the broadcasting of TV channels - most often in DTH.

- DBS (Direct Broadcast Satellite) - direct radio and television services. They are used, but here we have a higher than standard frequency.

- DTH (Direct to Home) - this is the most commonly used service for broadcasting TV content to end users.

- MSS (Mobile Satellite Services) - provision of satellite connection to mobile objects.

- Amateur radio connections - are carried out by satellites in low or strongly elliptical orbit.

- GPS (Global Positioning System) - a network of mid-orbit satellites that position each object on earth.

- Meteorological satellites - meteorological satellites such as METEOSAT, NOOA for monitoring weather and atmospheric conditions and the earth. 
- Radio determination satellite service RDSS - used in aviation to accurately determine the location, speed or other parameters of objects.

- RNSS (Radio navigation satellite systems) - Radio navigation satellite services - used in the maritime industry to determine the time and location of the vessel.

- Military satellites - a network of satellites used entirely by the military.

Satellites, especially in low and medium orbit, are often grouped into LEO/MEO constellations. This approach allows the transmission of large volumes of data, which gives them a significant advantage. In addition, they have minimal latency (connection delay), unlike GEO satellites. This huge amount of data, which is expected to be available to end users around 2021, will significantly reduce the cost of 1 Mbps. This will significantly increase the consumption and demand for these services, given the fact that now a large part of the population does not have access to the Internet (especially in Africa), given the lack of communication infrastructure and more potential users will request the services of LEO satellites operators. As we know, SES Astra launched O3B satellites for this purpose - to power the Other 3 Billion - the other 3 billion people without Internet access. However, this was not such a successful attempt, given the unaffordable price of this satellite technology. This is the goal of LEO operators - to provide quality and cheap internet service to a large number of people in remote areas. It will also be the technological revolution that Musk, Bezos and Branson will provide by joining this space race.

\section{The commercial start of low and medium orbit satellites}

Commercial constellations that are not in geostationary orbit date back to the late 1990s [11]. ICO, Teledesic, Skybridge, Celestri, Iridium and Globalstar are pioneers in this field. In fact, many of them have not had a successful design and application, with the exception of Iridium and GlobalStar, whose low-orbit satellite networks are still active today and successfully generate high revenue for organizations. Teledesic included at that time in its plans the launch of 288 satellites in low orbit. Even if we compare with today's constellations, such a realization seems quite ambitious and imaginary. Teledesic, Celestri and Sky Bridge target mainly fixed services, while other companies are targeting mobile - especially in areas and regions that are not well covered by conventional cellular networks.

More than 100 companies have officially announced their desire to build loworbit constellations, but only a few of them we would define as market leaders and feasible. These are-O3B SES, One Web, Kepler Communications, Telesat, Amazon Project, Kuiper and Space X Starlink.

\section{Review of LEO projects and integration with Teleport operators}

One of the significant projects is that of SES - O3B, which is a network of satellites in medium Earth orbit. The service was launched in September 2014 and within 12 
months 12 satellites were launched into orbit with a total capacity of $192 \mathrm{Gbps}$ and 40 active clients. The company succeeds thanks to its idea of targeting markets such as the maritime industry, oil companies, mobile operators (satellite access to provide a mobile network) and the government sector. The combination of strong beam, flexibility, low delay time, powerful and affordable terminals, give a good perspective of the constellations in low and medium Earth orbit.

Of the five companies that are leaders in the LEO market, only two of them announce the integration of Teleport services in their business. There is certainly a trend for the development of ground station operators, given the need for them in the construction of these networks. Kepler, for example, does not need ground stations, but only one Teleport is needed to start the project. However, if Kepler manages to launch all 140 satellites that are part of their plans, they will certainly have to expand their Teleport partners to process all data sets. The Canadian company already has two nano satellites in polar orbit, and new 16 are expected in 2020. Each nano satellite weighs about $10 \mathrm{~kg}$, has 2 to 3 years of life, and their huge advantage is that they are built much faster and cheaper, compared to most GEO and even LEO satellites. They also contain software-based radio channels for orbit correction. Kepler focuses on two market verticals - one is companies that need to transfer larger data sets, but which are not sensitive to time lag. Supported speeds range from $40 \mathrm{Mbps}$ download to $20 \mathrm{Mbps}$ upload. The second market niche is IoT (Internet of Things) solutions that require real-time communication and data transmission [12]. Different techniques are proposed to cope with latency and energy consumption when collecting and processing end user data. For example, the used fog technique allows improving the QoS and decreasing communication cost [13]. Keppler is actively involved in joint developments for mobile terminals with market leaders such as Kymeta and Phasor, supporting speeds of up to $15 \mathrm{Mbps}$ download and $5 \mathrm{Mbps}$ upload.

One Web has already launched 6 satellites in February 2019 and is conducting tests for Full HD video broadcasting, as well as data transfer up to $400 \mathrm{Mbps}$ with a signal latency of less than 40 milliseconds, as well as easy transfer of information between individual satellites. New 30 satellites are expected soon to be part of the big constellation. However, One Web is heavily dependent on ground stations and operators to deploy their entire project. The company analyses and selects Teleport stations with enough physical space for antenna colocation, redundant power supply, as well as high-capacity optical connectivity to provide the satellite network. One Web practically relies on ground stations that have a good technical infrastructure, as well as the availability of space for colocation of antennas. However, the goal is not only to host the equipment, but also for their partners to become their distributors of satellite capacity. The total value of the One Web system is about $\$ 3.5$ billion.

SES, on the other hand, is in the process of developing their second project in the O3B series - O3B MPower. It will have about 4,000 beams directing to only one satellite, unlike the original O3B project, which has 10 beams. The final client 
terminal would reach $10 \mathrm{Gbps}$, with the first satellite expected to be launched in 2021 . Their strategy is to use their own Teleports, as well as external ones, which can support the huge capacities that the network should provide to end users.

Telesat has 16 active satellites and a network approval of 117. Most market experts consider this to be one of the most efficient constellations in terms of capacity and speed. The satellites will operate in a polar orbit at an altitude of about $1000 \mathrm{~km}$ from the earth's surface and an inclined orbit at $2348 \mathrm{~km}$. The total allocated capacity is $4 \mathrm{Ghz}$ in $\mathrm{KU}$ band. The plan includes building a network of about 50 teleport ground stations to build the transmission. The antennas that are part of the satellites will be routing to secure areas that do not have sufficient Internet access. One of the guarantees for this project is that it is funded by the Canadian government, which makes it one of the safest in the field for successful implementation.

Amazon announced this year its intention to launch 3,236 satellites that will aim to provide broadband Internet access to millions of households in the United States and around the world. Meanwhile, in 2018, the company announced the AWS Ground Station initiative, which will be integrated with the Lockheed Martin - Verge system. In this way, each user has the opportunity to download data from low-orbit satellites through Amazon's network of ground stations. This is certainly a good opportunity for new profits, given the trend of launching thousands of satellites. Amazon's concept is to provide free access to space for users who can't build their own technology infrastructure to support the service. In addition, they aim to integrate the digital ground station with other cloud solutions that are already part of their portfolio.

Starlink is the latest project, which we will also review briefly. For us, this is one of Elon Musk's most ambitious plans. Space X's plans are similar to other companies - to supply the Internet inaccessible and unconnected to the global network regions of the world. The final phase of the project includes the presence of a total of 12,000 satellites (according to the latest information). In 2020,60 satellites were regularly launched from a Falcon 9 rocket based on Space X. The fact is that 4 of the satellites were lost by the company in an attempt to avoid a collision. The service will launch mainly in the United States and Canada, and according to information from the company, consumers will be able to take advantage of the service through a small pizza scale terminal at a price of about $\$ 200$. Starlink has demanded that regulators be able to activate up to 1 million antennas of this type.

Finally, in addition to the fact that it is difficult to predict what share of these projects will be completed successfully and to what extent it is appropriate to invest in this area, we will add that LEOSAT - a company that was one of the serious competitors, announced that it will cease operations. Their goal was to build a constellation of 108 satellites to provide connectivity to customers in the oil industry, maritime business, and large corporations with special business operations in areas with low network infrastructure through inter-satellite links. 


\section{Impact on the technological ecosystem}

Some aspects of the impact of the elements of the satellite telecommunications chain with each other and on the rest of the technological infrastructure are [14]:

- Teleport infrastructure - in practice Teleports, which should be part of LEO networks, must meet different requirements from standard GEO satellites.

The focus here is no longer the equatorial arch, but the whole horizon. The antennas should be more than one, given the lack of a fixed position of the satellite relative to the ground, and they must have good visibility to the sky to ensure reliable communication. An important point when choosing the location is the lack of other frequency interference, such as $5 \mathrm{G}$ transmitters. In the case of building KA band constellations, the climate is extremely important - for example, heavy rains could completely cut off the connection to the ground station. Antennas are the most visible part of the infrastructure, but they are not the most important. The operator is obliged to provide the necessary boxes/closets for hosting the equipment, as well as the necessary cooling and requirements for fire safety, security of access, disaster recovery and others to support the services. However, in most cases this leads to the need for additional investment in order to meet all the requirements of satellite operators. It should be noted that the collocation of such equipment can be easily performed by existing engineering staff and does not require the appointment of new people, given that the service functions smoothly after its configuration and periodic hardware checks are required.

- The satellite ecosystem - experts in the satellite business have differing opinions about the success of the constellations in low and medium Earth orbit.

It is not yet clear what the impact will be on existing geostationary networks. It is not yet clear whether the price to the end user will be lower, but these are the market expectations. One of the main obstacles is that the equipment that is currently in active use has not yet been adapted to the new LEO technologies. Market leaders such as Intellian are also developing so-called multi-orbit antennas that can work simultaneously in different orbits. In addition, antennas are being developed to operate in different frequency bands, which gives users a significant advantage in choosing the most appropriate service for them. At the same time, the antennas support high-speed data transmission. Recently, a new innovative product from Kymeta appeared - a flat terminal/flat panel, which can be easily installed on any surface. Unfortunately, the price of this type of hardware is not yet affordable for ordinary users. This is because the antennas have to be motorized, given the constant movement of the satellite, which makes the product more expensive. In our opinion, this will be one of the main new niches for business development. Companies that create equipment to support these technologies will earn new revenue. However, the question remains whether the cost of integrating these services will be more 
affordable given the still expensive equipment needed to build such telecommunications connectivity.

Mobility is the main vertical to which the new constellations in low and medium Earth orbits are directed. However, this involves many challenges. For example, an airplane needs two parabolic or one electronically directed antenna to provide a connection. However, their installation is associated with problems regarding their weight and the location of the fuselage where to place it. In addition, obtaining a certificate takes a long time, which means removing the machine from operation, and this leads to serious financial losses for airlines. The same is true for the maritime industry, but on a smaller scale and correspondingly smaller losses.

\section{LEO satellites for monitoring the earth's surface}

In recent years, there has been an increase in services provided through satellites that are in low Earth orbit and aim to capture the earth's surface [15]. Most of them operate at an altitude of $500-900 \mathrm{~km}$, gravitating around the earth in the so-called solar-synchronous orbit. Their position is significantly lower than geostationary ones, because given their distance of about $36,000 \mathrm{~km}$, it would be impossible to take highquality images that would be suitable for analysis in various fields. In 2008, about 150 satellites were allocated in space, which transmit $10 \mathrm{Tbps}$ of data per day. In 2018 , their number increases to 680 , which already require additional ground infrastructure. Teleport operators will also have the opportunity to expand their business, given this trend.

According to leading analysts, about $40 \%$ of satellites in low Earth orbit will perform ground-based imaging missions, and this is another trend that leads to new opportunities for development (Fig. 2).

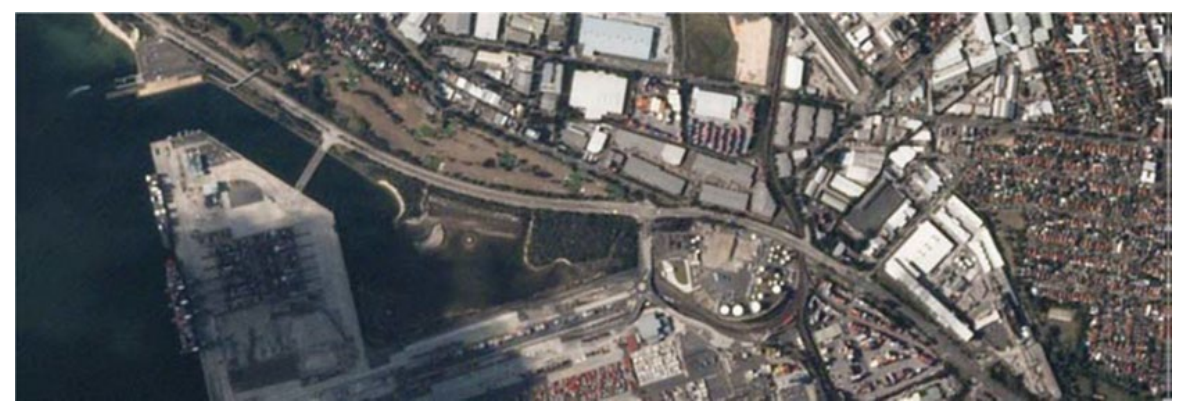

Fig. 2. Photo of the earth from LEO satellite

Some of the main areas in which satellites find applications are agriculture, atmospheric monitoring and land surface change, water basins, disaster and accident prevention, such as fires or volcanic activity and climate change. The Copernicus project includes several satellites called Sentinel, which are funded by the European Space Agency and the European Commission (Fig. 3). 


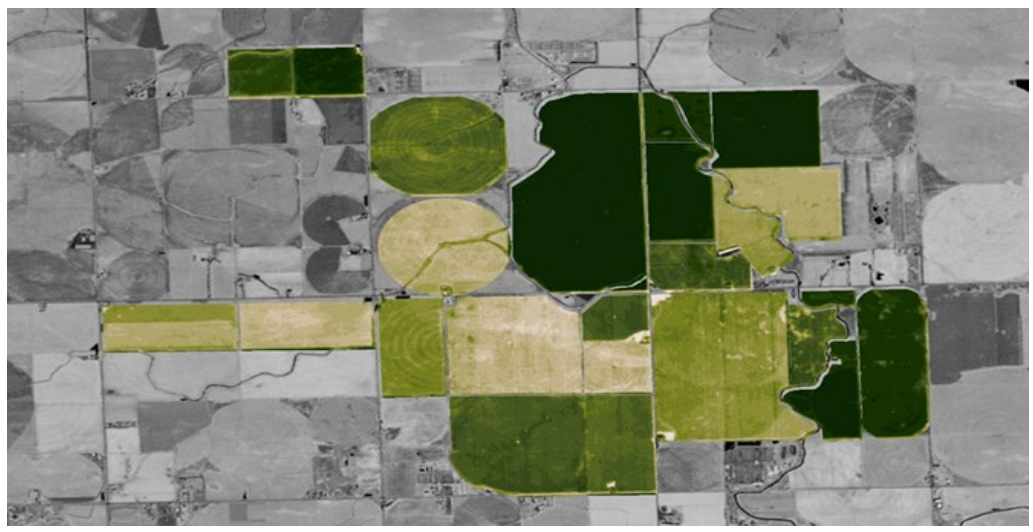

Fig. 3. Photo with life index data from Planet Lab

The name was given in 2012, and the main mission is the operational monitoring of the concentration of gases in the atmosphere. The main measurements are made of ozone, nitrogen dioxide, sulfur dioxide and others. At the same time, Sentinel constantly captures a large part of the earth's surface, providing completely free access to data sets in a common HUB. This allows everyone to access photos from a desired location. A leading private company on the other hand is PlanetLab, which has a constellation in low orbit, which provides data from different geographical locations, but with a higher frequency (on a daily basis), as well as with a higher resolution, allowing for more accurate survey of the specific geographical point.

\section{Conclusion}

After Arthur Clarke, who first proposed the idea of communications satellites, the satellite industry and telecommunications experienced unprecedented growth. Huge volumes of data of any type can now be transferred with minimal delay and to virtually anywhere in the world. The reception and processing of this data can now be done on smaller but more powerful devices. Some of them, such as mobile phones, have become an integral part of our daily lives. In many cases, users can communicate directly with satellites. There have also been offers to buy/launch miniature satellites, even for the needs of an individual or for a single purpose.

\section{References}

1. Bleazard, G.B.: Introducing Satellite Communications, New York: Halted Press (1985).

2. Blonstein, L.: Communications Satellites: Technology of Space Communications. New York: J. Wiley, (1987).

3. Lee, I., Lee. K.: The Internet of Things (IoT): Applications, investments, and challenges for enterprises. Business Horizons 58(4), 431-440 (2015). 
4. Liao, B., Ali, Y., Nazir, S., He, L., Khan, H. U.: Security analysis of IoT devices by using mobile computing: A systematic literature review. IEEE Access, vol. 8, pp. 120331120350, (2020), doi: 10.1109/ACCESS.2020.3006358.

5. Garvanov, I., Kabakchiev, H., Behar, V., Garvanova, M., Iyinbor, R.: On the modeling of innovative navigation systems. In: Shishkov B. (eds) Business Modeling and Software Design. Lecture Notes in Business Information Processing, vol. 356 (2019), https://doi.org/10.1007/978-3-030-24854-3_23.

6. Garvanov, I., Kabakchiev, C., Garvanova, M., Shishkov, B.: Vehicle navigation in urban environments: Overcoming GPS shadows. In: B. Shishkov (Ed.), International Conference on Telecommunications and Remote Sensing - ICTRS'18, pp. 95-98 (2018), doi: https://doi.org/10.1145/3278161.3278175.

7. Burrows, W. E.: This New Ocean: The story of the first space age. New York: The modern Library, (1999).

8. Types of Satellite Orbits, https://iasmania.com/types-of-satellite-orbits/

9. Global VSAT Training, https://gvftraining.org/

10. VIVACOM / Satellite services web site, https://www.vivacom.bg/bg/business/uslugi-zadostavchici/satelitni-uslugi

11. Eutelsat official web site, https://www.eutelsat.com/en/home.html

12. Yoshinov, R., Kulikov, I., Zhukova, N.: Methods of composing hierarchical knowledge graphs of telecommunication networks. Problems of Engineering Cybernetics and Robotics 72, 69-78 (2020).

13. Vodyaho, A., Yoshinov, R., Zhukova, N., Thaw, A.M., Ahmed Saddam A.: Fog oriented model for data collection in the networks of mobile devices. In: 2020 IEEE 10th International Conference on Intelligent Systems (IS), pp. 421-425 (2020), doi: 10.1109/IS48319.2020.9200138.

14. International Communications Union, https://www.itu.int/en/Pages/default.aspx

15. Intelsat, http://www.intelsat.com 